\title{
Sarcoidosis induced interstitial nephritis. A case series with literature review
}

\author{
Huzaif Qaisar, Shreya Gor, Anmol Cheema, Alireza Amirpour, Avais Masud, Diana Currasmartin, Mohammad A. Hossain* and Arif Asif \\ Department of Medicine, Jersey Shore University Medical Center, Hackensack Meridian Health, Neptune, NJ 07753, USA
}

\begin{abstract}
Sarcoidosis is an idiopathic granulomatous disease with organ dependent unpredictable prognosis. Renal dysfunction secondary to sarcoidosis is rare and often due to multiple etiologies including hypercalcemia, hypercalciuria and interstitial granulomatosis. Absence of interstitial granulomas does not preclude the diagnosis of sarcoid interstitial nephritis. Timely diagnosis and initiation of steroids can prevent further progression and can improve the renal function. We present 3 cases of sarcoidosis interstitial nephritis with and without granulomas in interstitium. All the cases mainly presented with generalized weakness and fatigue, who were found to have AKI and hypercalcemia. Our first case was thought to have AKI secondary to hypercalcemia, which resolved with iv hydration, and the patient was discharged. He was readmitted in 3 months for AKI and pancytopenia and was found to have sarcoidosis induced nephritis without interstitial granulomas on renal biopsy. His renal function improved significantly after treatment with steroids. The other two cases had an early diagnosis of sarcoid interstitial nephritis with granulomas demonstrated on renal biopsy. Renal function improved significantly with steroids. In this article, we have discussed our 3 cases along with review of literature between 2013 to 2018, with the primary aim of enforcing the need for early recognition and treatment of sarcoid induced nephritis with steroids which could significantly reduce both short and long-term morbidity and mortality associated with the disease.
\end{abstract}

\section{Introduction}

Sarcoidosis is an idiopathic multisystem granulomatous disease that has been postulated to be autoimmune etiology [1]. Incidence and prevalence of sarcoidosis is variable depending on geographic region and has been reported as 10 per 100,000 per year [2-4]. Highest incidence has been noted in the African American population [5]. Diagnosis is mostly dependent on a biopsy of an involved organ showing non-caseating granulomas as there is no reliable test to rule in or rule out this disease [6]. Lungs are most commonly involved but extra pulmonary manifestations have also been reported. Renal manifestation secondary to sarcoidosis are mostly due to hypercalcemia and hypercalciuria leading to nephrocalcinosis but clinically apparent renal failure is less common [1]. We present 3 cases of renal failure secondary to sarcoidosis and all them had features of interstitial nephritis on renal biopsy. Apart from the cases presented, we reviewed all cases of sarcoidosis induced interstitial nephritis reported from 2013 to 2018. There was a total of 13 case reports found that were reviewed. Sarcoidosis induced interstitial nephritis without granuloma formation has not been reported in the past 5 years which was observed in one of our cases. Once this condition is diagnosed, steroids with and without immunosuppressive agents play important role in the prognosis of renal sarcoidosis to prevent end stage renal disease (ESRD) $[1,6]$.

\section{Case 1}

An 81-year-old Caucasian male was referred from the outpatient clinic for evaluation of hypercalcemia with a serum calcium level of $13.7 \mathrm{mg} / \mathrm{dl}$ that was performed to evaluate his generalized weakness and worsening confusion for 1-2 weeks. His past medical history was significant for bladder cancer status post-surgery. He was suspected to have malignancy related hypercalcemia. As a work up for malignancy, his CT scan abdomen and pelvis showed para aortic and left inguinal lymphadenopathy. His initial laboratory investigation revealed elevated serum creatinine of $2.07 \mathrm{mg} / \mathrm{dl}$ (Table 1). The patient's acute kidney injury (AKI) was presumed to be secondary to hypercalcemia which improved with aggressive hydration. Fine needle aspiration of the left inguinal lymph node was inconclusive due to inadequate sampling $\mathrm{He}$ was discharged after resolution of hypercalcemia and AKI. Outpatient bone marrow biopsy for evaluation of pancytopenia revealed noncaseating granulomas. Three months after initial hospitalization, patient was sent to emergency department again from primary care office for AKI and pancytopenia. On admission, he subjectively had generalized weakness although his physical examination was unremarkable, including vital signs. He was worked up for vasculitis and multiple myeloma which came back negative. Infectious causes of granulomatous disease including hepatitis panel, histoplasmosis, and tuberculosis were negative. Renal ultrasound showed bilateral increased echogenicity. Patient underwent renal biopsy due to suspicion for sarcoidosis induced interstitial nephritis. His calcium level improved to $9 \mathrm{mg} / \mathrm{dL}$ but creatinine remained persistently elevated (5.03 to 5.43) despite aggressive hydration. On patient request, he was discharged with plan for starting steroids as an outpatient. The renal biopsy showed tubular degenerative changes, interstitial edema, mild diffuse interstitial inflammation with multifocal mild to moderate lymphocytic tubulitis, mild tubular atrophy and interstitial fibrosis,

${ }^{\star}$ Correspondence to: Mohammad A Hossain MD, Core faculty and Assistant Professor of Medicine, Jersey Shore University Medical Center, Hackensack Meridian Health, Hackensack-Meridian School of Medicine at Seton Hall 1945 Route 33, Neptune, New Jersey, USA, E-mail: mohammad.hossain@ hackensackmeridian.org

Key words: sarcoidosis, granulomatous disease, interstitial nephritis

Received: January 08, 2019; Accepted: January 11, 2019; Published: January 18 2019 
Table 1. Summary of Laboratory results

\begin{tabular}{|c|c|c|c|c|c|}
\hline \multirow{2}{*}{ Laboratory data } & \multicolumn{2}{|c|}{ Case 1} & \multirow{2}{*}{ Case 2} & \multirow[t]{2}{*}{ Case 3} & \multirow{2}{*}{ Reference Range } \\
\hline & First admission & Second admission & & & \\
\hline BUN & 28 & 79 & 57 & 55 & $5-25 \mathrm{mg} / \mathrm{dL}$ \\
\hline Creatinine & 2.07 & 5.23 & 4.0 & 6 & $0.62-1.24 \mathrm{mg} / \mathrm{dL}$ \\
\hline GFR & 31 & 11 & & & $>60$ \\
\hline Calcium & 14.0 & 14.0 & 14.3 & 14.6 & $8.5-10.5 \mathrm{mg} / \mathrm{dL}$ \\
\hline Albumin & 3.6 & 2.4 & 3.6 & 3.7 & $3.5-5.0 \mathrm{~g} / \mathrm{dL}$ \\
\hline Hemoglobin & 13.1 & 9.9 & 11.7 & 11.5 & $12-17.5 \mathrm{gm} / \mathrm{dL}$ \\
\hline WBC & 5,4 & 3.2 & 5.8 & 10.3 & $4.5-11.0 \mathrm{~K} / \mathrm{uL}$ \\
\hline Platelet count & 214 & 115 & 265 & 333 & $140-450 \mathrm{~K} / \mathrm{uL}$ \\
\hline SPEP & $-*$ & & $-*$ & & \\
\hline PSA & 9.76 & & 0.3 & & $0.0-4.0 \mathrm{ng} / \mathrm{mL}$ \\
\hline ALP & 249 & 176 & 47 & & $38-126 \mathrm{iU} / \mathrm{L}$ \\
\hline 25- $\mathrm{OH}$ vitamin $\mathrm{D}$ & 65 & 27 & & & $30-100 \mathrm{ng} / \mathrm{mL}$ \\
\hline 1-25 dihydroxy vitamin $\mathrm{D}$ & 152 & 18.0 & & & $19.9-79.3 \mathrm{pg} / \mathrm{mL}$ \\
\hline ACE level & & 92 & 166 & 111.5 & $9-67 \mathrm{U} / \mathrm{L}$ \\
\hline PTH & 8.8 & 16.8 & 6 & & $12-88 \mathrm{pg} / \mathrm{mL}$ \\
\hline PTHrp & 4.3 & & $<0.3$ & & $0.0-2.3 \mathrm{pmol} / \mathrm{L}$ \\
\hline ANA & & -0.62 & & & $<0.90$ \\
\hline ANCA & & - & & & \\
\hline Urine total protein & & & 1330 & & $5-100 \mathrm{mg} / 24$ hours \\
\hline CEA level. & 2.1 & & & & $0-10 \mathrm{ng} / \mathrm{mL}$ \\
\hline AFP & $<2.5$ & & & & $0-9.0 \mathrm{ng} / \mathrm{mL}$ \\
\hline
\end{tabular}

Empty cells mean no data available. BUN: Blood urea nitrogen. GFR: Glomerular filtration rate. WBC: White blood cells. SPEP: Serum protein electrophoresis. PSA: Prostate specific antigen. ALP: Alkaline phosphatase. 25 OH Vitamin D: 25 hydroxy vitamin D. ACE: Angiotensinogen converting enzyme. PTH: Parathyroid hormone. PTHrp: PTH related peptide. ANA: Anti-nuclear Antibody. ANCA: Anti-neutrophil cytoplasmic antibody. CEA: Carcino-embryonic antigen. AFP: Alpha feto protein. * Negative result

and microscopic tubulopapillary lesion, suggestive of sarcoidosis induced interstitial nephritis. After treatment with steroids creatinine significantly improved $(3.03 \mathrm{mg} / \mathrm{dL})$ on repeat blood work one month after discharge.

\section{Case 2}

A 62 years-old male with no significant past medical history, initially presented with sleepiness, weakness, polyuria, low-grade fever for 2-3 weeks and $100 \mathrm{lbs}$. weight loss over a 2-year period. Physical examination was unremarkable. Laboratory investigations revealed elevated blood urea nitrogen, serum creatinine level and hypercalcemia (Table 1). He was admitted due to AKI in the setting of hypercalcemia with the differential diagnosis of possible malignancy versus parathyroid disease. Angiotensin converting enzyme (ACE) level was elevated 166 $\mathrm{U} / \mathrm{L}$ (9-67), parathyroid hormone (PTH) intact $107 \mathrm{pg} / \mathrm{mL}$ (14.0-72.0), PTH related peptide $<0.3 \mathrm{pmol} / \mathrm{L}(0.0-1.5)$, urine total protein 1330 $\mathrm{mg} / 24$ hours (5-100), and serum protein electrophoresis revealed no monoclonal peak. Chest x-ray and computed tomography showed mediastinal lymphadenopathy. Patient underwent Para tracheal lymph node biopsy for further evaluation of possible lymphoma or myeloma, which showed small non-necrotizing granulomas consistent with sarcoidosis. A renal biopsy was then performed that demonstrated granulomatous interstitial nephritis, moderate tubular atrophy, interstitial fibrosis, moderate arterio and arteriolosclerosis with hyalinosis. The patient was diagnosed with sarcoidosis induced bone marrow infiltration and interstitial nephritis. He was started on steroids which resulted in significant improvement of renal function.

\section{Case 3}

A 30 years-old male without significant past medical history presented with fatigue, back pain, polydipsia and polyuria for 4-5 months. He also reported a 50 lbs. weight loss over 5 months. Physical examination was unremarkable except multiple palpable lymph nodes in axilla and inguinal region. Laboratory investigation revealed hypercalcemia, elevated BUN and serum creatinine levels (Table 1). He was admitted for acute renal failure. Computed Tomography (CT) chest, abdomen and pelvis showed extensive mediastinal, mesenteric, retroperitoneal lymphadenopathy. Inguinal lymph node biopsy was obtained which showed confluent epitheloid granulomas consistent with sarcoidosis. Auramine and AFB stains were negative for mycobacteria. GMS stain was negative for fungal pathogens. Angiotensin converting enzyme (ACE) level was elevated 111.7 U/L (967). Further workup ruled out hematologic malignancies and bone scan showed no skeletal mass. Renal biopsy was performed which showed severe acute and chronic interstitial nephritis with focal granulomatous features. Patient's renal function significantly improved after treatment with steroids.

\section{Discussion}

Sarcoidosis is an idiopathic multisystem autoimmune inflammation that is most commonly seen in the African American population with the lungs most commonly involved [2,3]. Hypercalcemia and hypercalciuria leading to direct tubular damage and nephrolithiasis play key role in its pathogenesis [5]. Renal dysfunction secondary to sarcoidosis is rare. Renal biopsy typically shows interstitial granulomas and reported to be found in 7 to $23 \%$ of cases when autopsies of patients with sarcoidosis induced kidney injury are performed [7-9]. Most cases of sarcoid interstitial nephritis have been reported with renal biopsy showing non-caseating granulomas and not even one case was reported without granulomas in kidney except for one of our cases where renal biopsy showed interstitial inflammation but no granuloma (Table 3) [10-22].

In our 3 cases of sarcoid induced interstitial nephritis, there were 2 cases with granulomas in kidney and bone marrow and the other case of already diagnosed sarcoidosis who presented with renal dysfunction but there were no granulomas seen on the kidney biopsy. 
All three had the common symptoms of weakness and fatigue and were found to have AKI and hypercalcemia. The first case primarily had granulomatous infiltration of the bone marrow but not the kidneys. He did have inguinal and para-aortic lymphadenopathy, but no other manifestations. His diagnosis was delayed. The second case had an early diagnosis and initiation of steroids compared to the previous case. Our third case was unusual with the predominant extra pulmonary manifestation of diffuse lymphadenopathy which raised a strong suspicion of lymphoma. Peripheral lymphadenopathy is common in younger patients and is seen in about $40 \%$ of patients with sarcoidosis. All our three cases had some form of lymphadenopathy. Most common form of lymphadenopathy seen is hilar and Para tracheal, present in almost $90 \%$ of patients $[23,24]$. However, our patient had mediastinal lymphadenopathy without hilar adenopathy which is very uncommon in sarcoidosis [25]. All our cases showed significant improvement in the renal function after treatment with steroids.

Apart from the cases presented, we reviewed all cases of sarcoidosis induced interstitial nephritis reported from 2013 to 2018. Thirteen cases were reviewed, 6 males and 7 females, with a mean age of 56 years. Most common symptom at presentation was weight loss followed by fatigue, malaise, fever, nausea, vomiting, lethargy and anorexia [10-22]. All patients presented with renal dysfunction with mean creatinine of $4.9 \mathrm{mg} / \mathrm{dL}$. Proteinuria was reported in 7 patients and was not documented in 3 cases. Angiotensin converting enzyme level (ACE) was reported to be elevated in 6 patients and was not documented in one patient (Table 2). Hypercalcemia was reported in 6 patients at the time of presentation with no documentation of calcium level in one case (Table 2). Most common extra renal manifestation include lungs followed by lymph node, salivary glands, bone marrow, spleen and brain respectively [10-22]. Mediastinal lymphadenopathy was present in $46.15 \%$ of cases (Table 2). Renal biopsy revealed granulomatous interstitial nephritis in all 13 cases. Steroids were started as initial treatment in all thirteen cases and creatinine was followed. Creatinine levels normalized in $38.4 \%$ cases $53.8 \%$ showed improvement in serum creatinine after initiating steroids [10-22].

There was one case reported of sarcoid induced interstitial nephritis with extra renal granuloma with eosinophilic tubulointerstitial nephritis on renal biopsy and granulomas on liver biopsy [23]. There was also a case series of 94 cases of patients with sarcoid granulomatous interstitial nephritis from 1955 to 2005 which showed a mean age 46.9 [1]. This study explained various etiologies of sarcoidosis induced kidney damage including glomerular, tubular and interstitial damage secondary to hypercalcemia, hypercalciuria and granulomatous inflammation leading to clinically apparent renal failure [1]. Steroids played an important role in improving the prognosis in all cases reported in this case series [1].

Taro Horino et al. [10] reported the case with constitutional symptoms in a patient with history of uveitis for 2 years and renal failure on presentation as compared to our patient with no ophthalmologic presentation. Sarcoidosis has variable presentation

Table 2. Summary of 13 Reported Cases of Sarcoid induced interstitial nephritis

\begin{tabular}{|c|c|c|c|c|c|c|}
\hline Source & $\begin{array}{c}\text { Age } \\
(y) / \operatorname{Sex}\end{array}$ & Creatinine (mg/dl) & Proteinuria* & $\begin{array}{c}\text { Elevated Serum } \\
\text { Calcium } \\
(>11 \mathrm{mg} / \mathrm{dl})\end{array}$ & $\begin{array}{c}\text { Serum } \\
\text { ACE }\end{array}$ & Mediastinal adenopathy \\
\hline Taro et al. [10] & $62 \mathrm{~F}$ & 4.56 & $+(1.4 \mathrm{~g} / \mathrm{g})$ & + & & + \\
\hline Amel et al. [11] & $35 \mathrm{~F}$ & 6.06 & $-(<0.5 \mathrm{~g} /$ day $)$ & - & + & - \\
\hline Hiroyuki et al. [12] & $60 \mathrm{~F}$ & 4.2 & & + & & - \\
\hline Varun et al. [13] & $66 \mathrm{M}$ & 5.4 & $+(1+)$ & - & & - \\
\hline Joana et al. [14] & $26 \mathrm{~F}$ & 2.0 & $+(1.03 \mathrm{~g} / 24 \mathrm{hrs})$ & + & + & + \\
\hline Hiroaki et al. [15] & $70 \mathrm{M}$ & 2.39 & $+(1+)$ & - & & - \\
\hline Nagaraja et al. [16] & $56 \mathrm{~F}$ & 3.0 & $-(0.24 \mathrm{~g} / 24 \mathrm{~h})$ & - & + & + \\
\hline Amel et al. [17] & $37 \mathrm{~F}$ & 5.4 & & + & + & - \\
\hline Tamires et al. [18] & $65 \mathrm{M}$ & 8.65 & $+(0.5 \mathrm{~g} / 1)$ & + & + & + \\
\hline Saika et al. [19] & $55 \mathrm{M}$ & 7.6 & & + & + & + \\
\hline Yoshinori et al. [20] & $77 \mathrm{~F}$ & 3.19 & $-(0.4 \mathrm{~g} / 24 \mathrm{~h})$ & - & & + \\
\hline Sharica et al. [21] & $44 \mathrm{M}$ & 3.42 & $+(1070 \mathrm{mg} / \mathrm{g})$ & + & & - \\
\hline Shinichiro et al. [22] & $79 \mathrm{M}$ & 7.97 & $+(2+)$ & - & & - \\
\hline
\end{tabular}

Empty cell means no data available. ACE: Angiotensin converting enzyme, * Data in uniform units for proteinuria not available.

Table 3. Summary of Renal Biopsy and Extra-renal manifestation in 13 Reported Cases

\begin{tabular}{|c|c|c|}
\hline Source & Granulomas on biopsy & Extrarenal manifestation \\
\hline Taro et al. $[10]$ & + \\
\hline Amel et al. $[11]$ & + & + \\
\hline Hiroyuki et al. $[12]$ & + & + \\
\hline Varun et al. $[13]$ & + & + \\
\hline Joana et al. $[14]$ & + & + \\
\hline Hiroaki et al. $[15]$ & + & + \\
\hline Nagaraja et al. $[16]$ & + & + \\
\hline Amel et al. $[17]$ & + & + \\
\hline Tamires et al. $[18]$ & + \\
\hline Saika et al. $[19]$ & + \\
\hline Yoshinori et al. $[20]$ & + \\
\hline Sharica et al. $[21]$ & + \\
\hline Shinichiro et al. $[22]$ & + \\
\hline
\end{tabular}

Empty cells means no data available 
and renal manifestation is rare. It can present with Lofgren syndrome that include arthralgia and erythema nodosum as seen in one of the cases found in which vasculitis and splenic nodules were shown as presentation of sarcoidosis along with salivary gland granulomas [11]. Parotid gland is the most common salivary gland involved that can present as painless bilateral cheek swelling or painful parotitis $[16,18]$. Hypercalcemia and elevated ACE levels are also helpful but not diagnostic as shown in less than $50 \%$ of the 13 cases studied. Bone marrow involvement is also not a common finding in sarcoidosis patients. Disturbance of renal functions with high suspicion of sarcoidosis should lead to renal biopsy as was performed in all cases studied. The findings of interstitial nephritis with granuloma formation was found in all except our case which showed no granulomas but presence of lymphocytic inflammation, tubulitis and tubular atrophy. Other renal biopsy findings may include glomeruli with focal ischemic tuft retractions and interstitial fibrosis [11-14]. Anti-glomerular basement membrane (GBM) antibodies should be tested as co-occurrence of Anti GBM antibodies in renal sarcoidosis was reported by in one of the cases reviewed in which no other light microscopic findings were found consistent with anti GBM antibodies [20]. Steroids are the main stay of treatment once the diagnosis is made. All our patients responded to steroids and renal function improved. Steroid sparing agents such as azathioprine can be used for those cases with gradual improvement to avoid the devastating side effects from long term steroid use $[11,16]$.

Since 2006 comprehensive review by Berliner et al. [1], many more cases have been reported. This is updated review in which 13 cases with enough reportable data were found and studied. All cases were presented with granuloma formation on kidney biopsy except for the one of our cases which did not have granulomas on renal biopsy. This is a unique finding from other cases with granulomas reported between 2013 and 2018.

\section{Conclusion}

Sarcoidosis induced interstitial nephritis is always a concern for internist and nephrologist but is often diagnosed late in the clinical course. Presence of interstitial granulomas is the most common finding of Sarcoid interstitial nephritis but it can be diagnosed without presence of granulomas in the right clinical setting as in our case. The absence of granulomas should not delay the treatment as it can lead to worse clinical prognosis with ESRD. This updated review of literature is to facilitate internists for prompt initiation of corticosteroids, early renal biopsy in a clinical situation concerning for sarcoidosis with renal failure and not exclude sarcoidosis induced interstitial nephritis even without the presence of granulomas.

\section{References}

1. Berliner AR, Haas M, Choi MJ (2006) Sarcoidosis: The Nephrologist's Perspective. $A m$ J Kidney Dis 48: 856 - 870. [Crossref]

2. Dumas O, Abramovitz L, Wiley AS, Cozier YC, Camargo CA Jr (2016) Epidemiology of Sarcoidosis in a Prospective Cohort Study of U.S. Women. Ann Am Thorac Soc 13: 67-71. [Crossref]

3. Rybicki BA, Major M, Popovich J Jr, Maliarik MJ, Iannuzzi MC (1997) Racial differences in sarcoidosis incidence: a 5 -year study in a health maintenance organization. Am J Epidemiol 145: 234-41. [Crossref]

4. Ungprasert P, Carmona EM, Utz JP, Ryu JH, Crowson CS, et al. (2016) Epidemiology of Sarcoidosis 1946-2013: A Population-Based Study. Mayo Clin Proc 91: 183-8. [Crossref]

5. Thomas KW, Hunninghake GW (2003). Sarcoidosis. JAMA 289: 3300-3303.

6. Iannuzzi MC, Rybicki BA, Teirstein AS (2007) Sarcoidosis. N Engl J Med 357: 2153 2165 .
7. Branson J, Park J (1954) Sarcoidosis hepatic involvement: Presentation of a case with fatal liver involvement, including autopsy findings and review of the evidence for sarcoid involvement of the liver as found in the literature. Ann Intern Med 40:111-145, 1954

8. Longcope W, Freiman D (1952) A study of sarcoidosis; Based on a combined investigation of 160 cases including 30 autopsies from The Johns Hopkins Hospital and Massachusetts.General Hospital. Medicine (Baltimore) 31: 1-132. [Crossref]

9. Ricker W, Clark M (1949) Sarcoidosis: A clinicopathologic review of 300 cases, including 22 autopsies. Am J Clin Pathol 19: 725-749. [Crossref]

10. Horino T, Matsumoto T, Inoue K, Ichii O, Terada Y (2018) A case of acute kidney injury caused by granulomatous interstitial nephritis associated with sarcoidosis. CEN Case Rep 7: 34-38. [Crossref]

11. Harzallah A, Kaaroud H, Boubaker K, Barbouch S, Goucha R, et al. (2017) Acute kidney injury with granulomatous interstitial nephritis and vasculitis revealing sarcoidosis. Saudi J Kidney Dis Transpl 28: 1157-1161. [Crossref]

12. Tsukada H, Shimizu H, Kaname S (2017) Bilateral nephromegaly due to isolated renal sarcoidosis. Clin Exp Nephrol 21: 169-170. [Crossref]

13. Agrawal V, Crisi GM, D'Agati VD, Freda BJ (2012) Renal sarcoidosis presenting as acute kidney injury with granulomatous interstitial nephritis and vasculitis. $\mathrm{Am} \mathrm{J}$ Kidney Dis 59: 303-8. [Crossref]

14. Rema J, Carvalho M, Vaz R, Fonseca M, Sampaio S, et al. (2014) Acute renal failure as a form of presentation of sarcoidosis in a young adult: a case report. J Med Case Rep 8: 274. [Crossref]

15. Kikuchi H, Mori T, Rai T, Uchida S (2015) Acute kidney injury caused by sarcoid granulomatous interstitial nephritis without extra renal manifestations. CEN Case Rep 4: 212-217.

16. Nagaraja P, Davies MR (2014) Granulomatous interstitial nephritis causing acute renal failure: a rare presenting feature of sarcoidosis. QJM 107: 467-9. [Crossref]

17. Ghani AA, Al Waheeb S, Al Homoud E (2011) Isolated sarcoid renal granulomatous tubulointerstitial disease. Saudi J Kidney Dis Transpl 22: 1208-10. [Crossref]

18. 18.Piraciaba TT, Balda CA, Moura LAR, Pereira CAC, Kirsztajn GM (2017) Granulomatous intersticial nephritis secondary to sarcoidosis. J Bras Nefrol 39: 473476. [Crossref]

19. Sharmeen S, Kalkan E, Yi C, Smith SD (2016) Granulomatous Interstitial Nephritis Presenting as Hypercalcemia and Nephrolithiasis. Case Rep Nephrol 4186086. [Crossref]

20. Osamu H, Kensuke J (2016) A case of acute kidney injury caused by granulomatous tubulointerstitial nephritis associated with sarcoidosis and concomitant presence of anti-glomerular basement membrane antibody. Sarcoidosis Vasc Diffuse Lung Dis 32: 368-71. [Crossref]

21. Brookins SM, Ormenisan C, Makary RF, Monteiro CI, James LR (2015) The bumpy lumpy kidney that dumped protein: granulomatous glomerular and tubulointerstitial nephritis. Kidney Int 88: 917. [Crossref]

22. Ikeda S, Hoshino T, Nakamura T (2013) A case of sarcoidosis with severe acute renal failure requiring dialysis. Clin Nephrol 82: 273-7. [Crossref]

23. Andreoli S, Kleiman M, Yum M, Bergstein J (1981) Acute eosinophilic interstitial nephritis and sarcoidosis. Arch Pathol Lab Med 105: 626-627. [Crossref]

24. Bajwa R, Amin A, Gandhi B, Mansur S, Amirpour A, et al. (2018) A simplified approach to the management of hypercalcemia. The Open Urology \& Nephrology Journal 11: 22-27.

25. Müller NL, Kullnig P, Miller RR (1989) The CT findings of pulmonary sarcoidosis: analysis of 25 patients. Am J Roentgenol 152: 1179-82. [Crossref]

26. Vagal AS, Shipley R, Meyer CA (2007) Radiological manifestations of sarcoidosis Clin Dermatol 25: 312-25.

Copyright: (C2019 Qaisar H. This is an open-access article distributed under the terms of the Creative Commons Attribution License, which permits unrestricted use, distribution, and reproduction in any medium, provided the original author and source are credited. 